\title{
Organspende - eine Ausnahmesituation im OP
}

\author{
$\square$ Frank Reis, Kai Nehammer
}

\section{Zusammenfassung}

Organspenden sind heute in jedem Krankenhaus mit Intensivstation und Operationsabteilung möglich und die Meldung potenzieller Organspender ist gesetzlich vorgeschrieben (Transplantationsgesetz). Die Deutsche Stiftung Organtransplantation (DSO) ist die bundesweite Koordinierungsstelle für Organspenden in Deutschland. Informationsdefizite über Hirntod und Organspende führen immer wieder zu Unsicherheiten im Umgang mit der Situation und $\mathrm{zu}$ scheinbaren Gewissenskonflikten. Die Koordinatoren der DSO organisieren den gesamten Prozess der Organspende vor Ort und sind Ansprechpartner für die Pflegenden im OP in der Akutsituation und für Fortbildungen.

\section{Organ Donation - an Exceptional Situation in the Operation Theatre}

Today organ donations can be done in any hospital with an intensive care unit and operation theatre. According to the transplantation law, the authorities must be notified of every potential organ donor. DSO (Deutsche Stiftung Organtransplantation) is the national German organ procurement organisation. Frequently, information deficits concerning brain death and organ donation lead to problems in handling the situation and ethical pseudo-conflicts. The DSO case managers in organ donation are responsible for organisation of the complete process of an organ donation in a given hospital, are the partners for OR nurses in the case of an organ donation and are responsible for education on this topic.

\section{Organspende -}

\section{eine Ausnahmesituation im OP}

Mehr als 12000 schwerkranke Patienten warten in Deutschland auf eine Organtransplantation. Leider herrscht in Deutschland ein sehr großer Mangel an transplantablen Organen, da sich zu wenige Menschen entscheiden, nach ihrem Tod mit einer Organspende anderen Menschen ein Weiterleben zu ermöglichen und sich auch zu wenige Krankenhäuser an der Organspende beteiligen. Der Tod auf der Warteliste ist deshalb in Deutschland eine bittere Realität: pro Jahr sterben weit über 1000 Menschen, weil nicht rechtzeitig ein Organ für sie verfügbar ist.

Organspenden sind heute in allen Krankenhäusern mit Intensivstationen und Operationsabteilungen möglich und gehören gemäß Transplantationsgesetz zum Versorgungsauftrag aller Kliniken.

Das ist noch zu wenig bekannt und führt dazu, dass nicht alle potenziellen Organspender an die DSO gemeldet werden, obwohl es eine gesetzliche Pflicht zur Mitwirkung an dieser Gemeinschaftsaufgabe gibt.

Der Weg zur Transplantation beginnt, wenn auf der Intensivstation eines Krankenhauses ein Patient mit einer schweren Hirnschädigung (z.B. einer Hirnblutung) Symptome des Hirntodes entwickelt und die Ärzte ihn als möglichen Spender der Deutschen Stiftung Organtransplantation (DSO) melden.

Die DSO ist die bundesweite Koordinierungsstelle für Organspenden in Deutsch- land, mit regionalen Organisationszentralen und -schwerpunkten in allen Bundesländern vertreten und bietet über Servicenummern rund um die Uhr Beratung für die Krankenhäuser an.

Zusätzlich kommt ein Koordinator der DSO ins Krankenhaus und übernimmt die Organisation aller weiteren Schritte der Organspende, beginnend mit der Hirntoddiagnostik durch erfahrene Ärzte nach den Richtlinien der Bundesärztekammer. Hirntod bedeutet, dass durch die Hirnschädigung oder -verletzung alle Gehirnfunktionen inklusive der Atemtätigkeit unwiederbringlich zerstört sind. Dieser Zustand darf nicht mit dem Wachkoma oder apallischen Syndrom verwechselt werden, bei dem eindeutig sichtbar noch Gehirnfunktionen vorhanden sind und sich deshalb jede Frage nach einer Organspende verbietet (Abb.1).

Die endgültige Feststellung des Hirntodes ist der Todeszeitpunkt des Patienten.

Eine Organentnahme kann grundsätzlich nur dann stattfinden, wenn es eine $\mathrm{Zu}-$ stimmung in mündlicher oder schriftlicher Form des Verstorbenen zu Lebzeiten gibt (z.B. Organspenderausweis oder Patiententestament) oder, wenn die Angehörigen im mutmaßlichen Willen des/ der Verstorbenen einer Organentnahme zustimmen.

Organspender sind heute nicht mehr nur junge Menschen, die an Kopfverletzungen nach Verkehrsunfällen sterben, sondern in der Mehrzahl Menschen im mittleren oder höheren Lebensalter, die an einer Hirnblutung oder anderen Hirnschädigungen (z.B. hypoxisches Hirnödem) versterben. Eine Altersbegrenzung bei der Frage nach einer Organspende gibt es nicht mehr. Alle Organe werden deshalb auf ihre Eignung für Transplantationen genau untersucht. 


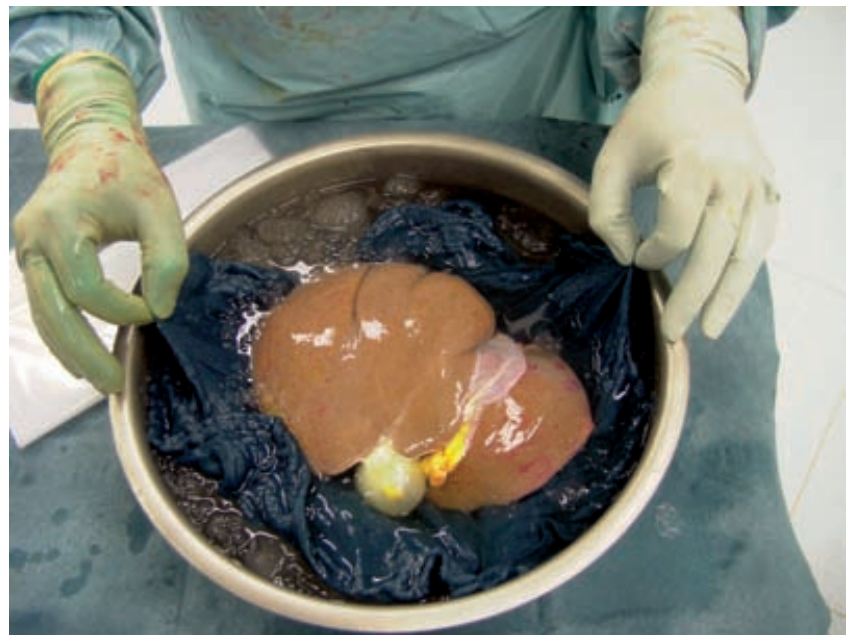

Abb. 1 Explantierte Leber.

Der DSO-Koordinator übermittelt die gesammelten medizinischen Daten an die Vermittlungsstelle Eurotransplant in Leiden/Holland. Dort werden anhand der Blutgruppe und der Gewebemerkmale entsprechend der Richtlinien der Bundesärztekammer die geeigneten Empfänger für die gespendeten Organe gesucht.

Danach beginnt die „heiße Phase“ der Organspende mit der Planung der Entnahmeoperation. In Absprache mit den lokalen OP-Koordinatoren und der Anästhesieabteilung legt der Koordinator einen OP-Zeitpunkt fest. Die Organe werden von erfahrenen Transplantationschirurgen entnommen, die in das Krankenhaus kommen, in dem sich der Organspender befindet. Die abdominale Organentnahme führt ein Team aus einem regionalen Transplantationszentrum in räumlicher Nähe des Krankenhauses durch. Herz und Lungen entnehmen Teams aus den Zentren, an die Eurotransplant die Organe vermittelt hat. Die Logistik der Teamtransporte ist eine der Hauptaufgaben des DSO-Koordinators.

Organtransplantationen sind die einzige oder überlegene Therapie bei allen Arten des irreversiblen Organversagens. Organspenden als Voraussetzung für Transplantationen werden in den Krankenhäusern aber immer noch nicht genügend als normaler Bestandteil der Arbeit gesehen. Dies betrifft auch die Pflegenden im OP, in deren Fachweiterbildung die Voraussetzungen für eine Organspende und der Ablauf von Organspenden zu wenig oder gar nicht behandelt werden. Dies führt manchmal dazu, dass in der Akutsituation aufgrund von Informationsdefiziten bei Pflegenden im OP Gewissenskonflikte auftreten, weil der Hirntod und seine Konsequenzen nicht vollständig verstanden wurden und nicht klar ist, dass Organspender definitiv verstorben sind, bevor sie in den OP gebracht werden. Der scheinbare Widerspruch eines schlagenden Herzens bei einem verstorbenen Menschen bereitet vielen Kopfzerbrechen. Auch die vom normalen operativen Vorgehen deutlich unterschiedliche chirurgische Technik ist ungewohnt, weil Gewebeschonung nur noch im Hinblick auf die zu entnehmenden Organe erforderlich ist. $\mathrm{Zu}$ wenig beachtet wird auch, dass eine Organentnahme den Willen des Verstorbenen, seine Organe zu spenden, umsetzt.

Da heute nur noch sehr selten Patienten während einer Operation versterben, ist das Miterleben des induzierten Herzstillstandes beim Durchspülen der Organe mit Konservierungslösungen und das

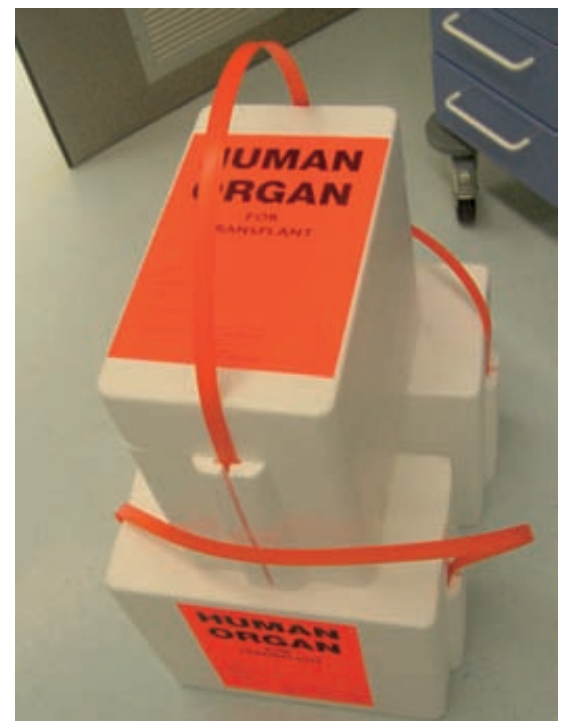

Abb.2 Organtransportboxen evtl. Beobachten einer Herzentnahme für Unvorbereitete möglicherweise ein schockierendes Erlebnis.

Die beste Vorbeugung gegen solche psychischen Belastungen sind Gespräche und Fortbildungen zu diesem Thema und eine Festlegung von Zuständigkeiten und Abläufen in hausinternen Ablaufplänen.

Der würdevolle Umgang mit dem Verstorbenen und der Respekt vor den trauernden Angehörigen bleiben in jeder Phase der Organspende oberstes Gebot für alle Beteiligten. Die DSO-Koordinatoren begleiten die Organspende bis zum Schluss und kümmern sich um die korrekte Versorgung des Leichnams. Innerhalb einer Woche berichten die Koordinatoren allen Beteiligten an einer Organspende in einem Brief über die Transplantationsergebnisse. Auch die Angehörigen erhalten auf Wunsch einen solchen Brief und werden regelmäßig zu psychologisch betreuten Treffen mit anderen Betroffenen eingeladen.

Die Koordinatoren der DSO sind jederzeit gerne bereit, in Krankenhäusern Informationsveranstaltungen für OP-Fachpflegende durchzuführen. Die zuständigen regionalen DSO-Organisationszentralen erfahren Sie im Internet unter www.dso.de, durch eine E-Mail an kh-info@dso.de oder unter Tel. 061023008280 .

Eine Kurzinformation mit Angaben zu den für eine Organentnahme benötigten Instrumenten und Geräten erhalten Sie bei den Verfassern.

\section{Frank Reis}

Koordinator in der Organspende Fachpfleger für Anästhesie und Intensivmedizin

\section{Dr. med. Kai Nehammer}

Koordinator in der Organspende Facharzt für Anästhesie, DEAA

DSO/Region Mitte

Adam-Karillion-Str. 23

D-55118 Mainz

DSO/Region Mitte

Home Office Saarbrücken

Schinkelstr. 16

66123 Saarbrücken 